\title{
A nonlinear convective model for radial stellar pulsations
}

\section{The physical description}

\author{
M.U. Feuchtinger \\ Institut für Astronomie der Universität Wien, Türkenschanzstraße 17, A-1180 Wien, Austria \\ Received November 6, 1998; accepted January 4, 1999
}

\begin{abstract}
The physical and numerical ingredients of the Vienna nonlinear model for radially pulsating stars are presented. Stellar pulsations are investigated by solving the full system of radiation hydrodynamics including a time-dependent formulation of turbulent convection. The numerical solution method comprises a state-of-the-art adaptive grid algorithm which provides a powerful tool for resolving and tracking the most important ionization zones. Several already published applications to pulsating stars in the classical instability strip demonstrate that the model is able to reproduce important observational properties like RR Lyrae light curves or double mode pulsations.
\end{abstract}

Key words: stars: variables — hydrodynamics — stars: oscillations - methods: numerical

\section{Introduction}

Due to extensive observational activities in the last decade we dispose of a huge amount of accurate experimental data about pulsating stars. In particular the microlensing projects OGLE (e.g. Kaluzny et al. 1998) and MACHO (e.g. Alcock et al. 1996, 1998) as by-products provide a large number of high-quality photometric data of radially pulsating stars (e.g. Cepheids, RR Lyrae, W Virginis, AGB stars), many of them newly discovered. From the theoretical point of view these data in a unique way constrain our theoretical models. We use this models to understand the physical processes acting in pulsating stars. Consequently, in order to benefit from the variety of detailed observations, high-quality computations of stellar pulsations are necessary in order to reproduce the observational facts with the required accuracy.

Send offprint requests to: M.U. Feuchtinger, Internet: FM@AMOK.ASTRO.UNIVIE.AC.AT
The aim of this paper, which is the first in a series dealing with nonlinear pulsation models, is to review the fundamental physical and numerical methods we employ to compute realistic models of radially oscillating stars. The philosophy behind the development of this model is twofold. On the one hand it is essential to consider all physical processes expected to be important in a pulsating envelope. Apart from fluid dynamics this encloses timedependent radiative transfer and time-dependent turbulent convection. Consequently a comprehensive description of stellar oscillations requires a "convective radiation hydrodynamical" picture. By that we mean that the nonlinear equations of radiation hydrodynamics including a model for convective energy transfer have to be solved for the case of radial stellar pulsations.

The numerical difficulties usually encountered when investigating such complex nonlinear systems under astrophysical conditions naturally lead to our second demand, namely to use appropriate numerical methods. Analogous to most astrophysical problems in fluid dynamics, stellar pulsation flows exhibit a number of complicated nonlinear features like shock waves or moving ionization fronts, often acting on a non-trivial hydrostatic stellar background structure. In this context the main numerical problem is the sufficient resolution of all important structures during the whole computation. The basic consequence is to use an adaptive grid algorithm which is able to track all important flow features continuously with high spatial resolution. This involves a number of implications for the formulation of the physical equations and the numerical methods adopted for solving the discretized system of convective radiation hydrodynamics.

The paper is divided into two main parts: Sect. 2 summarizes all physical ingredients of the model and Sect. 3 is devoted to the numerical method of solution. Section 4 closes the paper with some general remarks about the applicability of the model. 


\section{Physical description}

In order to describe the nonlinear behavior of pulsating stars we assume the stellar plasma to consist of three components, namely gas, radiation and turbulent convection. The evolution of each component is governed by respective conservation laws and coupling terms between these equations account for their interaction. The resulting set of coupled equations called the system of convective radiation hydrodynamics (CRHD) consists of 7 nonlinear partial differential equations which have to be solved under appropriate initial and boundary conditions. All equations are written in spherical symmetry, meaning that each physical quantity is considered to be a function of radius $r$ and time $t$. Consequently we are restricted to radial pulsations.

\subsection{Gas dynamics}

The dynamics of the stellar gas is governed by three conservation laws for mass (continuity equation, Eq. (2) in Table 1), momentum (equation of motion, Eq. (3) in Table 1) and internal energy (gas energy equation, Eq. (4) in Table 1). The independent variables are the gas density $\rho$, the gas velocity $u$ and the gas internal energy $e$. In contrast to most nonlinear pulsation models we use an equation for the internal energy instead of the total energy equation. A detailed discussion of implications and consequences for stellar pulsation models is given in Feuchtinger \& Dorfi (1994, 1997). Additionally, as we consider selfgravitating flows, we solve the Poisson equation for the gravitational field. This is done by analytically integrating the Poisson equation in spherical symmetry yielding the gravitational force which is considered as a source term on the right-hand side of the equation of motion. The remaining integration of the inner mass $m_{\mathrm{r}}$ over the density structure is performed by solving Eq. (1) in Table 1, where we employ $m_{\mathrm{r}}$ as the independent variable.

Further details concerning the equations of hydrodynamics can be found e.g. in Mihalas \& Mihalas (1984, Chapter 7).

\subsection{Radiative transfer}

Time-dependent radiative transfer is considered in the grey approximation by solving the first two frequencyintegrated moment equations of the radiation field representing energy conservation (radiation energy equation, Eq. (5) in Table 1) and momentum conservation (radiation momentum equation, Eq. (6) in Table 1). As independent variables we use the zeroth moment of the radiation field $J$ and the first moment of the radiation field $H$, which are proportional to the radiative energy density and the radiative flux, respectively (e.g. Mihalas \& Mihalas 1984, page 337$)$.
A special remark concerns the dynamical terms in the radiation momentum equation. These terms account for time-dependent effects in the radiation field where the light travel time becomes comparable to the dynamical time scale of the problem (e.g. traveling light fronts). Such phenomena usually are not encountered in typical stellar pulsation environments and consequently these terms can be neglected.

In contrast to that the dynamical terms in the radiation energy equation are important whenever the ratio $u / c$ becomes comparable to the ratio $\lambda_{\mathrm{p}} / l_{\text {hyd }}$ with $\lambda_{\mathrm{p}}$ and $l_{\text {hyd }}$ denoting the photon mean free path and the typical hydrodynamical length scale of the problem (Mihalas \& Mihalas 1984, page 457). In this case (known as the dynamical diffusion regime) the advection of radiation energy by the moving fluid becomes important and such situations are likely to be encountered in stellar pulsation flows. In fact test calculations carried out with and without including the dynamical terms in the radiation energy equation exhibit differences in the pulsation properties like pulsation amplitudes and light curve shapes. Detailed results and further discussions will be presented in a separate paper.

\subsection{Convective transfer}

Radiation hydrodynamical models allow a self-consistent description of pulsating envelopes as long as convection is negligible. This means that a radiative pulsating envelope is determined completely by specifying a set of stellar parameters (mass, luminosity, effective temperature and chemical composition). However, it turned out that several properties of classically pulsating stars (Cepheids, RR Lyrae) like the pulsation amplitudes, the cool edge of the instability strip or double mode pulsations (Kolláth et al. 1998) cannot be explained with purely radiative models. In fact energy transfer by turbulent convection plays an essential role in the physics of stellar oscillations and therefore cannot be neglected (e.g. Yecko et al. 1998).

Due to the lack of a self-consistent convection theory the inclusion of turbulent convection into the radiation hydrodynamical model introduces a number of free parameters. In principle these parameters cannot be determined from theoretical considerations, but have to be chosen in order to fit observational constraints. In that sense a self-consistent modelling of stellar pulsations must await considerable progress in turbulence theory.

In the past decades a number of time-dependent one-dimensional models of turbulent convection have been developed. Most of these models are based on the Boussinesq-approximation which assumes the eddy gas to be incompressible except for buoyancy effects. Turbulent motions are considered as fluctuations of the mean hydrodynamic field and this assumption is used to linearize the equations in the fluctuating parts (see Baker 1987 for an overview). In the context of stellar pulsations the 
Table 1. Equations of convective radiation hydrodynamics in differential form. $D / D t$ denotes the comoving or substantial derivative with respect to time. Refer to Table 6 for a comprehensive list of symbols

$$
\begin{array}{ll}
\hline \text { Gas dynamics } & \\
\frac{\partial m_{\mathrm{r}}}{\partial r}=4 \pi r^{2} \rho & \text { (1) } \text { integrated Poisson equation } \\
\frac{D \rho}{D t}+\rho \nabla \cdot u=0 & \text { (2) continuity equation } \\
\frac{D(\rho u)}{D t}+\rho u \nabla \cdot u=-\frac{\partial P}{\partial r}-\frac{G m_{r} \rho}{r^{2}}+\frac{4 \pi}{c} \kappa \rho H-\frac{\partial P_{\mathrm{t}}}{\partial r}+U_{\mathrm{Q}} & \text { (3) equation of motion } \\
\frac{D(\rho e)}{D t}+\rho e \nabla \cdot u=-P \nabla \cdot u+4 \pi \kappa \rho(J-S)-\nabla \cdot j_{w}-\rho\left(S_{\bar{\omega}}-\tilde{S}_{\bar{\omega}}-D_{\mathrm{rad}}\right) & \text { (4) internal energy equation } \\
\hline \text { Radiative transfer }
\end{array}
$$$$
\begin{array}{ll}
\frac{D J}{D t}+J \nabla \cdot u=-c \nabla \cdot H-K \nabla \cdot u+(3 K-J) \frac{u}{r}-c \kappa \rho(J-S) & \text { (5) radiation energy equation } \\
\frac{D H}{D t}+H \nabla \cdot u=-c \frac{\partial K}{\partial r}-(3 K-J) \frac{c}{r}-H \frac{\partial u}{\partial r}-c \kappa \rho H & \text { (7) radiation momentum equation }
\end{array}
$$

Turbulent convection

$$
\begin{aligned}
& \frac{D(\rho \bar{\omega})}{D t}+\rho \bar{\omega} \nabla \cdot u=\rho\left(S_{\bar{\omega}}-\tilde{S}_{\bar{\omega}}-D_{\mathrm{rad}}\right)-\nabla \cdot j_{t}-P_{\mathrm{t}} \nabla \cdot u+E_{\mathrm{Q}} \\
& \text { (8) turbulent kinetic energy equation } \\
& S_{\bar{\omega}}=\frac{T}{P} \nabla_{\mathrm{s}} \frac{\partial P}{\partial r} \Pi \\
& \text { (9) turbulent driving through buoyancy forces } \\
& \Pi=\sqrt{\frac{2}{3}} \frac{w}{T} \bar{\omega}^{1 / 2} F_{L}\left[-\sqrt{\frac{3}{2}} \frac{\alpha_{\mathrm{s}} \Lambda T}{w} \frac{\partial s}{\partial r}\right] \\
& F_{\mathrm{L}}(x)= \begin{cases}x & |x|<1 \\
1 & |x| \geq 1\end{cases} \\
& \frac{\partial s}{\partial r}=\frac{1}{T} \frac{\partial e}{\partial r}-\frac{P}{\rho^{2} T} \frac{\partial \rho}{\partial r}=-\frac{c_{\mathrm{P}}}{H_{\mathrm{p}}}\left(\nabla-\nabla_{\mathrm{s}}\right) \\
& \tilde{S}_{\bar{\omega}}=c_{\mathrm{D}} \frac{\bar{\omega}^{3 / 2}}{\Lambda} \\
& D_{\mathrm{rad}}=\frac{\bar{\omega}}{\tau_{\mathrm{rad}}} \\
& \tau_{\mathrm{rad}}=\frac{c_{\mathrm{p}} \kappa \rho^{2} \Lambda^{2}}{4 \sigma T^{3} \gamma_{\mathrm{R}}^{2}} \\
& j_{\mathrm{t}}=\alpha_{\mathrm{t}} \rho \Lambda \bar{\omega}^{1 / 2} \frac{\partial \bar{\omega}}{\partial r} \\
& P_{\mathrm{t}}=\frac{2}{3} \rho \bar{\omega} \\
& E_{\mathrm{Q}}=\frac{4}{3} \mu_{\mathrm{Q}}\left\{\frac{\partial u}{\partial r}-\frac{u}{r}\right\}^{2} \\
& U_{\mathrm{Q}}=\frac{1}{r^{3}} \frac{\partial}{\partial r}\left\{\frac{4}{3} \mu_{\mathrm{Q}} r^{3}\left[\frac{\partial u}{\partial r}-\frac{u}{r}\right]\right\} \\
& \mu_{\mathrm{Q}}=\alpha_{\mu} \rho \Lambda \bar{\omega}^{1 / 2} \\
& \Lambda=\alpha_{\mathrm{ML}} H_{\mathrm{P}}^{\text {stat }} \\
& H_{\mathrm{P}}^{\text {stat }}=\frac{r^{2} P}{G m_{\mathrm{r}} \rho}
\end{aligned}
$$


majority of investigations use the convection model of Stellingwerf (1982a) to compute nonlinear limit cycle solutions for RR Lyrae and Cepheid pulsations (Stellingwerf 1982b; Gehmeyr 1992; Bono \& Stellingwerf 1994; Bono et al. 1997; Bono et al. 1998).

For the nonlinear pulsation model presented in this paper we use the one-equation model of time-dependent turbulent convection proposed by Kuhfuß (1986) in the version of Wuchterl \& Feuchtinger (1998). Although the approach is similar to the Stellingwerf-model there are some important differences discussed in Kuhfuß(1987) and Gehmeyr \& Winkler (1992). Recently a similar model was used for linear and nonlinear Cepheid pulsations by Yecko et al. (1998) and Kolláth et al. (1998).

The basic equation (Eq. (8) in Table 1) of the Kuhfuß model is a conservation law for the turbulent kinetic energy density $\bar{\omega}$, which serves as the independent variable of the turbulent field. The essential term is the turbulent driving through buoyancy forces $\left(S_{\bar{\omega}}\right)$. The basic quantity entering $S_{\bar{\omega}}$ is the entropy gradient $\partial s / \partial r$ which is related to the Schwarzschild criterion by Eq. (12) (Table 1 ). Note that $S_{\bar{\omega}}$ can be evaluated even if the Schwarzschild criterion indicates convective stability. This means that we do not have to neglect damping buoyancy forces as e.g. done in the Stellingwerf model (Stellingwerf 1982a). An important technical advantage of the adopted formulation for $S_{\bar{\omega}}$ is that we do not have to use a seed for the turbulence field.

Another term important for stellar pulsations is the energy dissipation $E_{\mathrm{Q}}$ through turbulent viscosity. A corresponding term accounting for the viscous momentum generation $\left(U_{\mathrm{Q}}\right)$ enters the equation of motion. While radiative pulsation models either exhibit too large oscillation amplitudes or have to include artificial dissipation effects (e.g. Feuchtinger \& Dorfi 1994) the turbulent viscosity provides a physically motivated dissipation, however, adjustable by a free parameter.

For further details about the convection model and its parameters we refer to Wuchterl (1995) and Wuchterl \& Feuchtinger (1998).

\subsection{Constitutive relations}

For closing the CRHD equations we need several constitutive relations. They specify the microscopic properties of the gas and the radiation field and are discussed in the following sections. Closure relations for the turbulent convection are implicitly included in the assumptions for modelling the different correlations terms and their physical meaning is outlined in Kuhfuß (1987).

\subsubsection{Equation of state}

The equation of state specifies pressure $P(\rho, e)$, temperature $T(\rho, e)$, isentropic temperature gradient $\nabla_{\mathbf{s}}(\rho, e)$ and specific heat at constant pressure $c_{\mathrm{P}}(\rho, e)$ of the gas with respect to the independent variables $\rho$ and $e$. Note that due to considering the radiation field as an extra component we employ the pure gas pressure instead of the total pressure in the equation of motion $(\partial P / \partial r)$, while the radiation pressure is treated as an extra term $\left(4 \pi / c \kappa_{H} \rho H\right)$. This difference is essential in the case of anisotropic radiation fields (variable Eddington factor, see Sect. 2.4.3) where the radiation pressure tensor does not reduce to a simple scalar pressure.

The equation of state is included in tabulated form and it is essential to use suitable interpolation schemes to obtain smooth surfaces and derivatives (Dorfi \& Feuchtinger 1995).

\subsubsection{Opacity}

The stellar opacity used in the form $\kappa=\kappa(\rho, T)$ is also included in tabulated form. As long as the pulsational driving is located at high optical depths it is appropriate to adopt the Rosseland mean of $\kappa$ which yields the correct momentum transfer in the diffusion limit. However, if e.g. extended atmospheres of pulsating AGB stars involving dust formation and radiation pressure driven winds are investigated, it is important to use other proper means of the opacity (Höfner et al. 1998). A detailed discussion about mean opacities and gray radiative transfer can be found in Mihalas \& Mihalas (1984, page 355).

\subsubsection{Eddington factor}

For the closure of the two radiation moment equations an equation of state for the radiation field by means of the Eddington factor $f_{\text {edd }}=J / K$ is specified. For the case of classical stellar pulsations (e.g. Cepheids, RR Lyrae) where the stellar atmosphere does not influence the pulsational behavior (Feuchtinger \& Dorfi 1994), the Eddington approximation $\left(f_{\text {edd }}=1 / 3\right)$ which accounts for anisotropies in the radiation field up to the first order, can be used. However, if the atmosphere is taken into account, as e.g. in Feuchtinger \& Dorfi (1994) or Höfner \& Dorfi (1997), it is important to employ a variable Eddington factor. In that case the radial structure of the Eddington factor is calculated by solving the time-independent grey radiative transfer equation (e.g. Balluch 1988).

\subsection{Source function}

For the source function $S(T)$ appearing in the energy coupling between matter and radiation we assume local thermodynamical equilibrium and neglect scattering. Consequently $S(T)$ is given by the Stefan-Boltzman law:

$S(T)=\frac{\sigma}{\pi} T^{4}$ 
Table 2. Equations of convective radiation hydrodynamics in integral form. $\mathrm{d} / \mathrm{d} t$ denotes the time derivative with respect to a moving coordinate system which is essential for discretization on an adaptive grid. $V=V(t)$ denotes a time-dependent volume and $\partial V$ its surface. Refer to Table 6 for a comprehensive list of symbols and to the text for further details

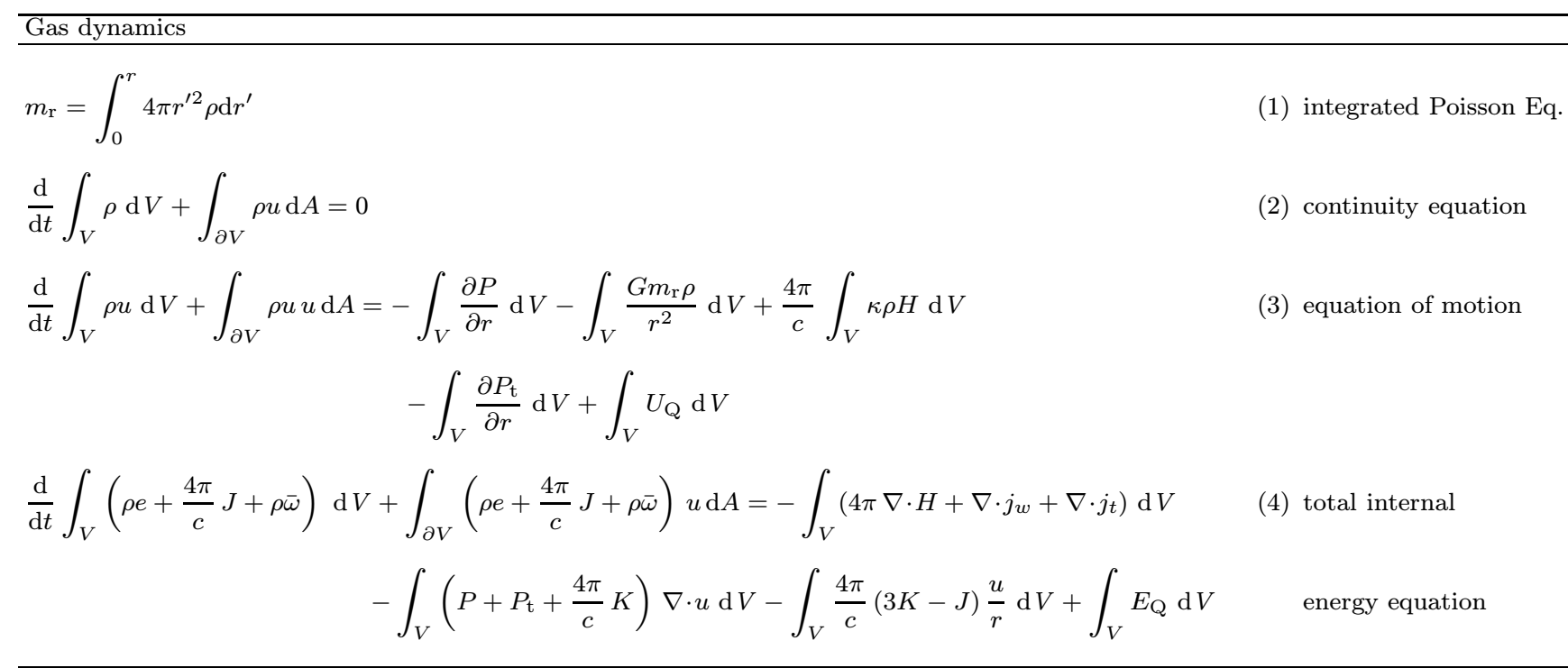

Radiative transfer

$$
\begin{aligned}
& \frac{\mathrm{d}}{\mathrm{d} t} \int_{V} J \mathrm{~d} V+\int_{\partial V} J u \mathrm{~d} A=-c \int_{V} \nabla \cdot H \mathrm{~d} V-\int_{V} K \nabla \cdot u+(3 K-J) \frac{u}{r} \mathrm{~d} V-c \int_{V} \kappa \rho(J-S) \mathrm{d} V \quad \text { (5) radiation energy Eq. } \\
& \frac{\mathrm{d}}{\mathrm{d} t} \int_{V} H \mathrm{~d} V+\int_{\partial V} H u \mathrm{~d} A=-c \int_{V} \frac{\partial K}{\partial r} \mathrm{~d} V-\int_{V}(3 K-J) \frac{c}{r} \mathrm{~d} V-\int_{V} H \frac{\partial u}{\partial r} \mathrm{~d} V-c \int_{V} \kappa \rho H \mathrm{~d} V \quad \text { (7) rad. momentum Eq. }
\end{aligned}
$$

Turbulent convection

$$
\frac{\mathrm{d}}{\mathrm{d} t} \int_{V} \rho \bar{\omega} \mathrm{d} V+\int_{\partial V} \rho \bar{\omega} u \mathrm{~d} A=\int_{V} \rho\left(S_{\bar{\omega}}-\tilde{S}_{\bar{\omega}}-D_{\mathrm{rad}}\right) \mathrm{d} V-\int_{V} \nabla \cdot j_{\mathrm{t}} \mathrm{d} V-\int_{V} P_{\mathrm{t}} \nabla \cdot u \mathrm{~d} V+\int_{V} E_{\mathrm{Q}} \mathrm{d} V \text { (8) turb. energy Eq. }
$$

\subsection{The model equations}

In Table 1 the complete set of model equations for gas dynamics, radiative transfer and turbulent convection in differential form are compiled. With regard to the numerical method of solution using a conservative volume discretization on an adaptive mesh (cf. Sect. 3), these equations have to be reformulated in conservation form and for a time-dependent coordinate system. This is done by integration over a time-dependent volume leading to the integral form of the CRHD equations as given in Table 2 . Note that due to numerical reasons we solve an equation for the total internal energy $(\rho e+4 \pi / c J+\rho \bar{\omega})$ instead of the gas internal energy equation. The corresponding total internal energy equation (Eq. (4) in Table 1) can be derived by adding the respective energy equations of gas, radiation and convection (Eqs. (4), (5) and (8) in Table 1).

\subsection{Boundary conditions}

For modelling stellar pulsation the CRHD equations are solved for a spherical shell representing the region of the star which is dominated and influenced by the oscillations.
Within the inner boundary located at $R_{\text {int }}$ we specify a rigid core which influences the model by its mass $M_{\text {int }}$ and luminosity $L_{\text {int }}$. Depending on the considered type of star the inner radius $R_{\text {int }}$ is constrained by two demands: First it has to be located above the zones where nuclear burning takes place, as we do not consider these effects. Secondly it has to be located deep enough in order to ensure that the eigenfunctions of the pulsations vanish at the inner boundary. Consequently for the mechanical inner boundary we assume the gas velocity to vanish $(u=0)$. An estimate for $R_{\text {int }}$ can be obtained from linear stability analysis. However, the independence of the nonlinear solution on $R_{\text {int }}$ has to be checked. For the turbulent convection at the inner boundary we assume the turbulent kinetic energy $\bar{\omega}$ to vanish which constrains the core to be radiative.

The outer boundary condition depends on the problem under consideration. If we are interested in classical stellar pulsations (e.g. Cepheids, RR Lyrae) which are strongly bound objects, oscillating weakly non-adiabatic and exhibiting negligible mass loss rates we use a Lagrangian outer boundary located above the photosphere. For that case the motion of the boundary is determined from the 
equation of motion. This requires that the medium outside the boundary is specified by an external pressure $P_{\text {ext }}$ which we assume to be constant. Note that $P_{\text {ext }}$ has to be sufficiently low compared with the pressure at the photosphere (Feuchtinger \& Dorfi 1994). Physically this means, that pulsational waves are almost perfectly reflected at the steep pressure decline of the atmosphere which is a good approximation for these stars. Consequently the penetration of waves into the stellar atmosphere are not considered which has no effect on the pulsational behavior (Feuchtinger \& Dorfi 1995). Again it has to be checked that the nonlinear solution is independent of $P_{\text {ext }}$. Usually we adopt a value of $P_{\text {phot }} / P_{\text {ext }}=30$ with $P_{\text {phot }}$ denoting the photospheric pressure.

If, on the other hand, pulsations of extended envelopes like AGB stars are to be investigated (which as a matter of fact is a really tough problem), because of the high mass loss rates expected in those stars, a Lagrangian boundary is not suitable. Pulsations are highly nonadiabatic meaning that transmission of mechanical energy into the atmosphere cannot be neglected. Consequently the outer boundary condition has to account both for a considerable expansion of the atmosphere and for the mass outflow generated by the pulsations. For further discussion we refer to Feuchtinger et al. (1993).

In addition to the mechanical outer boundary conditions a condition for the radiation field must be specified. Here we assume the radiative flux to have only an outward component leading to

$J=\frac{1}{\bar{\mu}} H$

with $\bar{\mu}$ denoting a quantity accounting for the geometry of the radiation field at the outer boundary. If we assume Eddington's approximation (cf. 2.4.3), $\bar{\mu}$ is given by $\bar{\mu}=$ $1 / 2$ while in the case of a variable Eddington factor $\bar{\mu}$ has to be determined from the radiation transport equation (Balluch 1988).

For the convective outer boundary we assume the gradient of the turbulent kinetic energy $\partial \bar{\omega} / \partial r$ to vanish which in principle allows a convective flux across the external radius. However, the outer boundary usually is located somewhere in the atmosphere where normally no convection occurs.

In Table 3 the complete set of boundary conditions for the case of classical stellar pulsations is summarized.

\subsection{Static equations and initial conditions}

In order to compute the nonlinear limit cycle evolution of a particular pulsating envelope we start from a hydrostatic configuration referred to as the initial model. Due to the implicit numerical method we use to solve the nonlinear equations (cf. Sect. 3) the structure of the initial model must be an exact solution of the full nonlinear equations. Numerically speaking the initial model has to lie within
Table 3. Boundary conditions for the case of classical stellar pulsations (see text for details)

\begin{tabular}{ll}
\hline Inner boundary & \\
\hline$r=R_{\text {int }}$ & core radius \\
$m_{\mathrm{r}}=M_{\text {int }}$ & core mass \\
$H=\frac{L_{\text {int }}}{\left(4 \pi R_{\text {int }}\right)^{2}}$ & core luminosity \\
$u=0$ & no motion \\
$\bar{\omega}=0$ & no convection \\
\hline Outer boundary & \\
\hline$r=R_{\text {ext }}$ & external radius \\
$P=P_{\text {ext }}$ & external pressure \\
$J=\frac{1}{\bar{\mu}} H$ & radiation field \\
$\frac{\partial \bar{\omega}}{\partial r}=0$ & no $\bar{\omega}$-gradient \\
\hline
\end{tabular}

the convergence radius of the implicit solution scheme. Consequently we use the hydrostatic and local limit of the nonlinear equations given in Table 1 to compute the structure of the initial model. This limit is obtained by omitting all time derivatives, setting the gas velocity identically to zero $(u \equiv 0)$, and neglecting turbulent pressure and overshooting. The remaining equations compiled in Table 4 yield a system of ordinary differential equations similar to the equations of stellar structure. Together with the constitutive relations given in Sect. 2.4 this system in principle can be solved by a forward integration.

However, for the case of pulsating envelopes without considering the stellar atmosphere the static equations can be further simplified by the following assumptions:

First we assume Eddington's approximation. Secondly we set $J=S$ which is true as long as layers are convectively stable. In this case the equations given in Table 4 reduce to the equations of stellar structure (e.g. in Kippenhahn \& Weigert 1990). If now, during the forward integration, a layer becomes convectively unstable (as determined by the Schwarzschild criterion) we replace the radiative temperature gradient in the radiation diffusion equation by the actual convective gradient, which can be determined from the static turbulent kinetic energy equation (Eq. (6) in Table 4). Analogous to the cubic equation of the mixing length theory a nonlinear equation for the actual temperature gradient can be derived (Wuchterl \& Feuchtinger 1998). 
Table 4. Hydrostatic and local limit of the convective radiation hydrodynamics equations given in Table 1. See text for details

$$
\begin{array}{ll}
\frac{\mathrm{d} m_{\mathrm{r}}}{\mathrm{d} r}-4 \pi r^{2} \rho=0 & \text { (1) integrated Poisson equation } \\
\frac{\partial P}{\partial r}+\frac{G m_{\mathrm{r}} \rho}{r^{2}}-\frac{4 \pi}{c} \kappa \rho H=0 & \text { (2) equation of motion } \\
\frac{\mathrm{d} j_{w}}{\mathrm{~d} r}+\frac{2}{r} j_{w}-4 \pi \kappa \rho(J-S)=0 & \text { (3) gas internal energy equation } \\
\frac{\mathrm{d} H}{\mathrm{~d} r}+\frac{2}{r} H+\kappa \rho(J-S)=0 & \text { (4) radiation energy equation } \\
\frac{\mathrm{d} K}{\mathrm{~d} r}+\frac{(3 K-J)}{r}+\kappa \rho H=0 & \text { (5) radiation momentum equation } \\
S_{\bar{\omega}}-\tilde{S}_{\bar{\omega}}-D_{\mathrm{rad}}=0 & \text { (6) turbulent kinetic energy equation }
\end{array}
$$

The above outlined method represents only an approximative solution of the static system compiled in Table 4. In particular at the transition regions between radiative and convective zones where both radiative and convective fluxes exhibit a nonvanishing divergence, the assumption $J=S$ is not fulfilled. However, in the majority of applications the solution lies well within the accuracy range necessary for the implicit solution method.

Due to practical reasons we start the integration at the photosphere where the initial values for mass, temperature, and luminosity are set to the stellar parameters of the model. Additionally we need the photosphere pressure which at first is set to an estimate. Beginning from the photosphere in a first step we perform an outward integration up to the desired external radius (cf. Sect. 2.7). At this point the outer boundary condition for the radiation field has to be matched and this relation is used to determine the photosphere pressure by iteration. After the atmosphere structure is computed in this way we continue by performing an inward integration from the photosphere down to the desired radius of the inner boundary condition.

In order to guarantee the initial model computed by forward integration to be hydrostatic with respect to the nonlinear equations, in a further step the nonlinear code is used to relax the model on the complete CRHD system. This is done by using the same numerical parameters as for the fully dynamical computation. A successful relaxation is done if the time step of the nonlinear evolution becomes large compared to the thermal time scale of the model, which normaly establishes after $10-20$ steps.

Even though the dynamical evolution is started with time steps lower than the dynamical time scale of the model, due to numerical dissipation a hydrostatic, but vibrationally unstable model usually remains hydrostatic, if the growth rates of the oscillations are small. In this case the pulsations are initiated by a velocity perturba- tion applied to the hydrostatic initial model. Depending to the problem under consideration the perturbation is taken stochastic or corresponding to the eigenfunction of the expected pulsation mode (Stobie 1969).

\section{Numerical solution}

The equations of convective radiation hydrodynamics given in Table 2 yield a system of 7 nonlinear coupled partial differential equations. Together with the initial and boundary condition discussed in Sects. 2.8 and 2.7 we obtain a initial-boundary-value problem which is solved numerically. The basic features of the employed numerical method are compiled in the following section, while detailed discussions can be found in the quoted papers.

\subsection{Adaptive grid}

For a continuous resolution and tracking of the driving zones and other moving flow features during the pulsation cycle we employ the adaptive grid algorithm developed by Dorfi \& Drury (1987). The basic concept is to distribute the available spatial grid points uniformly in arc-length along the graphs of the important physical variables. This is done by solving an additional equation (i.e. the adaptive grid equation) simultaneously with the physical equations. The major input to the grid equation is a set of physical quantities named marker functions, which control the grid distribution. This set has to be chosen according to the investigated problem. For stellar pulsations the set of marker functions given in Table 5 turned out to be appropriate for resolving and tracking all important physical quantities. In particular we found the isentropic temperature gradient $\nabla_{\mathrm{s}}$ to be very useful in order to resolve the HeII ionization zone responsible for the driving in Cepheids and RR Lyrae stars. Each marker function can be weighted separately in 
Table 5. Marker functions and weights adopted in the adaptive grid equation for the case of classical stellar pulsations

\begin{tabular}{lll}
\hline quantity & weight & \\
\hline$\rho$ & 1 & gas density \\
$e$ & 1 & gas internal energy \\
$\kappa \rho$ & 1 & opacity \\
$\nabla_{\mathrm{s}}$ & 5 & isentropic temperature gradient \\
\hline
\end{tabular}

order to account for different scales and the corresponding weight factors are also given in Table 5. Further information about the adaptive grid equation in the context of stellar pulsation calculations can be found in Feuchtinger \& Dorfi $(1994,1997)$.

\subsection{Discretization}

By adding the adaptive grid equation to the CRHD system (cf. Table 2) we end up with a system of 8 nonlinear partial differential equations. These equations are discretized in space and time by the method given in Tscharnutter \& Winkler (1979) and Winkler \& Norman (1986), which implies that the equations are formulated in conservation form. The employed volume discretization guarantees that global conservation of quantities like mass, momentum or internal energy, as prescribed by the differential equations, becomes an analytical property of the discretized equations.

Advection terms are treated by the monotonic scheme of van Leer (1977) which is $2^{\text {nd }}$ order in space. For the discretization of the time derivatives we adopt $2^{\text {nd }}$ order centered differences which requires an implicit method of solution (Winkler \& Norman 1986).

The resulting nonlinear algebraic system of discretized equations is solved implicitly by means of a multidimensional Newton-Raphson method. It is important to note that in contrast to explicit solution methods the timestep of the numerical solution is determined only by accuracy considerations and is not explicitly limited by extra relations like the Courrant-Friedrichs-Lewy condition. For further details we refer to Dorfi \& Feuchtinger (1995).

\subsection{Artificial viscosity}

In order to treat shock waves and other discontinuities by the finite difference method reviewed in the last section we use the artificial tensor viscosity developed by Tscharnutter \& Winkler (1979). In this context it is important to guarantee that the physical properties of the model are independent of the artificial viscosity. This requires the respective viscous length scale to be lower than the typical physical length scales of the problem. In the case of classically pulsating stars the important length scale is the pressure scale hight of the hydrostatic background structure which in the relevant regions is of the order of $10^{-1}-10^{-3}$ times the local radius (depending on the particular type of star and the position within the envelope). Therefore we chose a viscous length scale of the order $10^{-4}$ times the local radius in order to prevent that the results depend on the artificial viscosity.

The radiative models in Feuchtinger \& Dorfi (1994) use an additional artificial viscosity to adjust the amplitude of the pulsations. In the case of our convective model this viscosity is replaced by the turbulent eddy viscosity which serves as a free but physically motivated parameter and is used to tune the pulsation amplitudes.

\section{Applications}

The major objective of the Vienna nonlinear pulsation approach is to provide a model for radial stellar pulsations which in principle can be applied to all types of spherically symmetric stellar oscillations.

For the case of classical RR Lyrae pulsations it has already been shown, that long standing problems like the Fourier phase discrepancy or the computation of stable double mode pulsations can be resolved by using the outlined method (Feuchtinger \& Dorfi 1997; Feuchtinger \& Dorfi 1998; Feuchtinger 1998). An application to radiative Cepheid pulsation has been demonstrated in Dorfi \& Feuchtinger (1995), even though further computations are necessary in order to corroborate the applicability to these objects.

Due to the high spatial resolution provided by the adaptive grid it is possible to include the stellar atmosphere into the pulsation model. In this case the oscillations of the underlying envelope induce high amplitude shock waves propagating down the steep density gradient. For classical stellar pulsations it is generally assumed that the influence of the atmosphere can be neglected and this assumption was corroborated by a nonlinear RR Lyrae model including the atmosphere in Feuchtinger \& Dorfi (1994). Further applications of the model including detailed investigations of Cepheids and RR Lyrae stars are in progress and will be published in forthcoming papers.

One major unsolved problem in stellar pulsation theory is the computation of AGB pulsations (e.g. Tuchman 1998; Höfner 1998). In contrast to classically pulsating stars characterized by weak nonadiabaticity and small convection zones, AGB envelopes are dominated by convective energy transfer and highly nonadiabatic pulsations. Consequently a nonvanishing amount of mechanical energy is deposited into the stellar atmosphere which in turn influences the pulsating envelope. The levitation of the atmosphere through the pulsations yields conditions where molecules and dust grains can form and the radiation pressure on these new opacity sources causes a stellar wind to develop (e.g. Fleischer et al. 1992). 
Table 6. List of symbols

\begin{tabular}{|c|c|c|}
\hline \multicolumn{3}{|r|}{ Gas dynamics } \\
\hline$r$ & $\mathrm{~cm}$ & radius \\
\hline$t$ & $\mathrm{~s}$ & time \\
\hline$m_{\mathrm{r}}$ & $\mathrm{g}$ & mass within radius $r$ \\
\hline$\rho$ & $\mathrm{g} \mathrm{cm}^{-3}$ & density \\
\hline$e$ & $\operatorname{erg~g}^{-1}$ & specific internal energy \\
\hline$u$ & $\mathrm{~cm} \mathrm{~s}^{-1}$ & velocity \\
\hline$s$ & $\operatorname{erg} g^{-1} K^{-1}$ & specific entropy \\
\hline$w$ & $\operatorname{erg~g}^{-1}$ & specific enthalpy \\
\hline$\nabla$ & 1 & $\partial \ln T / \partial \ln P$ \\
\hline$P$ & dyn $\mathrm{cm}^{-2}$ & pressure \\
\hline$T$ & $\mathrm{~K}$ & temperature \\
\hline$\nabla_{\mathrm{s}}$ & 1 & isentropic temperature gradient \\
\hline$c_{\mathrm{P}}$ & $\operatorname{erg~g}^{-1} \mathrm{~K}^{-1}$ & specific heat at constant pressure \\
\hline$c_{\mathrm{S}}$ & $\mathrm{cm} \mathrm{s}^{-1}$ & speed of sound \\
\hline$\kappa$ & $\mathrm{cm}^{2} \mathrm{~g}^{-1}$ & opacity \\
\hline$S$ & $\operatorname{erg~cm}{ }^{-2} \mathrm{~s}^{-1}$ & source function \\
\hline$H_{\mathrm{P}}$ & $\mathrm{cm}$ & pressure scale hight \\
\hline & & Radiative transfer \\
\hline
\end{tabular}

$J \quad \mathrm{erg} \mathrm{cm}^{-2} \mathrm{~s}^{-1}$ zeroth moment of the radiation field

$H \quad \mathrm{erg} \mathrm{cm}^{-2} \mathrm{~s}^{-1}$ first moment of the radiation field

$K \quad \mathrm{erg} \mathrm{cm}^{-2} \mathrm{~s}^{-1}$ second moment of the radiation field

$f_{\text {edd }} 1$

$\begin{array}{ll}\bar{\mu} & 1\end{array}$

Eddington factor

geometry factor at the outer boundary

Turbulent convection

\section{$\bar{\omega} \quad \operatorname{erg~g}^{-1}$}

$\Lambda \quad \mathrm{cm}$

$u_{\mathrm{c}} \quad \mathrm{cm} \mathrm{s}^{-1}$

$S_{\bar{\omega}} \quad \operatorname{erg~g}^{-1} \mathrm{~s}^{-1}$

$\tilde{S}_{\bar{\omega}} \quad \operatorname{erg~g}^{-1} \mathrm{~s}^{-1}$

$\Pi \quad \mathrm{cm}^{3} \mathrm{~s}^{-3} \mathrm{~K}^{-1}$

$D_{\text {rad }} \operatorname{erg~g}^{-1} \mathrm{~s}^{-1}$

$\tau_{\text {rad }} \mathrm{s}$

$j_{w} \quad \mathrm{erg} \mathrm{cm}^{-2} \mathrm{~s}^{-1}$

$j_{\mathrm{t}} \quad \mathrm{erg} \mathrm{cm}^{-2} \mathrm{~s}^{-1}$

$P_{\mathrm{t}} \quad$ dyn $\mathrm{cm}^{-2}$

$\mu_{\mathrm{Q}} \quad \mathrm{cm}^{2} \mathrm{~s}^{-1}$

$E_{\mathrm{Q}} \quad \operatorname{erg~cm}^{-3} \mathrm{~s}^{-1}$

$U_{\mathrm{Q}} \mathrm{g} \mathrm{cm}^{-2} \mathrm{~s}^{-2}$

$\alpha_{\mathrm{ML}} 1$

$\alpha_{\mathrm{s}} \quad 1$

$\begin{array}{ll}c_{\mathrm{D}} & 1\end{array}$

$\begin{array}{ll}\alpha_{\mu} & 1\end{array}$

$\begin{array}{ll}\alpha_{\mathrm{t}} & 1\end{array}$

$\gamma_{\mathrm{rad}} 1$
Consequently a pulsation model applicable to AGB stars must be capable of including the stellar atmosphere with its complicated physical processes like timedependent dust formation, propagating shock fronts and radiation pressure driven winds. That this is possible with the method presented in this paper has been demonstrated by Höfner \& Dorfi (1997) who calculated the detailed structure of AGB atmospheres where the pulsations of the underlying star is simulated by a moving piston.

The remaining problem is to compute these pulsations, which mainly seems to be caused by an accuracy deficit. In contrast to classical pulsations AGB stars are gravitationally weakly bound. Energetical considerations reveal the total energy of AGB stars to be small compared with the internal energy, while e.g. for Cepheids or RR Lyrae stars the total energy always is of the order of the internal energy. Additionally the mass weighted mean of $\Gamma_{1}$ taken over a typical AGB envelope lies only slightly above the critical value of $4 / 3$ indicating dynamical instability. Numerically speaking small errors in the internal energy as produced by discretization or advection errors can be quite large when compared with the total energy. An amplification of such errors finally leads to a dynamical instability caused by numerical errors. As a consequence the main problem is to solve the nonlinear equations with sufficient accuracy.

Finally we want to draw attention to completely different astrophysical problems like evolution of supernova remnants (Dorfi 1994), formation of gaseous planets (Wuchterl 1993) and protostellar collapse calculations (Wuchterl 1998), where the same physical and numerical philosophy as reviewed in this paper has been employed successfully.

Acknowledgements. This work is supported by the Fonds zur Förderung der wissenschaftlichen Forschung ( $F W F)$ under project number S7305-AST.

\section{References}

Alcock C., et al., 1996, AJ 111, 1146

Alcock C., et al., 1998, ApJ 492, 190

Baker N., 1987, in: Physical Processes in Comets, Stars and Active Galaxies, Meyer-Hofmeister E., Thomas H.C. and Hillebrandt W. (eds.). Springer, Berlin, p. 105-124

Balluch M., 1988, A\&A 200, 58

Bono G., Caputo F., Castellani V., Marconi M., 1996, ApJ 471, L33

Bono G., Caputo F., Castellani V., Marconi M., 1997, A\&AS 121,327

Bono G., Caputo F., Castellani V., Marconi M., 1998 (in press)

Bono G., Stellingwerf R.F., 1994, ApJS 93, 233

Dorfi E.A., 1994, ApJS 90, 841

Dorfi E.A., Drury L.O'C., 1987, J. Comp. Phys. 69, 175

Dorfi E.A., Feuchtinger M.U., 1995, Comp. Phys. Comm. 89, 69

Feuchtinger M.U., 1998, A\&A 337, L29

Feuchtinger M.U., Dorfi E.A., 1994, A\&A 291, 225 
Feuchtinger M.U., Dorfi E.A., 1997, A\&A 322, 817

Feuchtinger M.U., Dorfi E.A., 1998, in: A half century of stellar pulsation interpretations: A tribute to Arthur N. Cox, PASPC 135, 297

Feuchtinger M.U., Dorfi E.A., Höfner S., 1993, A\&A 273, 513

Fleischer A.J., Gauger A., Sedlmayr E., 1992, A\&A 266, 321

Gehmeyr M., Winkler K.H.A., 1992, A\&A 253, 92

Höfner S., Dorfi E.A., 1997, A\&A 319, 648

Höfner S., Jørgensen U.G., Loidl R., Aringer B., 1999, A\&A 341

Höfner S., 1999, in: Asymptotic Giant Branch stars, Proc. IAU Symp. 191, Le Bertre T., Lebre A., Waelkens C. (eds.), ASP Conf. Ser. (in press)

Kaluzny J., Kubiak M., Szymanski M., Udalski A., Krzeminski W., Mateo M., 1997, A\&AS 125, 343

Kippenhahn R., Weigert A., 1990, Stellar structure and evolution. Springer, Berlin

Kolláth Z., Beaulieu J.P., Buchler J.R., Yecko P., 1998, ApJ 502, L55

Kuhfuß R., 1986, A\&A 160, 116

Kuhfuß R., 1987, "Ein Modell für zeitabhängige Konvektion in Sternen", PhD-Thesis, TU München
Mihalas D., Mihalas B.W., 1984, Foundations of Radiation Hydrodynamics. Oxford University Press, New York

Stellingwerf R.F., 1982a, ApJ 262, 330

Stellingwerf R.F., 1982b, ApJ 262, 339

Stobie R.S., 1969, MNRAS 144, 461

Tscharnutter W.M., Winkler K.-H.A., 1979, Comp. Phys. Comm. 18, 171

Tuchman Y., 1999, in: Asymptotic Giant Branch stars, Proc. IAU Symp. 191, Le Bertre T., Lebre A., Waelkens C. (eds.), ASP Conf. Ser. (in press)

van Leer B., 1977, J. Comp. Phys. 23, 276

Winkler K.-H.A., Norman M.L., 1986, in: Astrophysical Radiation Hydrodynamics, Winkler K.-H. and Norman M.L. (eds.), NATO-ASI Series C 188. D. Reidel, Dordrecht, p. 223

Wuchterl G., 1993, Icarus 106, 323

Wuchterl G., 1995, Comp. Phys. Comm. 89, 119

Wuchterl G., 1998, AGM Abstracts 14, B11

Wuchterl G., Feuchtinger M.U., 1999, A\&A 341

Yecko P.A., Kolláth Z., Buchler J.R., 1998, A\&A 336, 553 